\title{
Panel backs pain drug studies with new safety checks
}

The wait may be over for drugmakers developing the first ever antibody-based painkillers. Despite safety concerns that led the US Food and Drug Administration (FDA) to temporarily halt nearly all trials involving these new agents-which target the nerve growth factor (NGF) proteinan advisory committee to the agency voted overwhelmingly last month to recommend allowing such studies to resume.

"There was clearly a recognition that this is a new class of drugs, and it could help people with all sorts of chronic pain for which there are no good therapies available," says Brendan Boyce, director of anatomic pathology at the University of Rochester Medical Center in upstate New York and a member of the FDA's arthritis advisory panel. "But, going forward, what they need

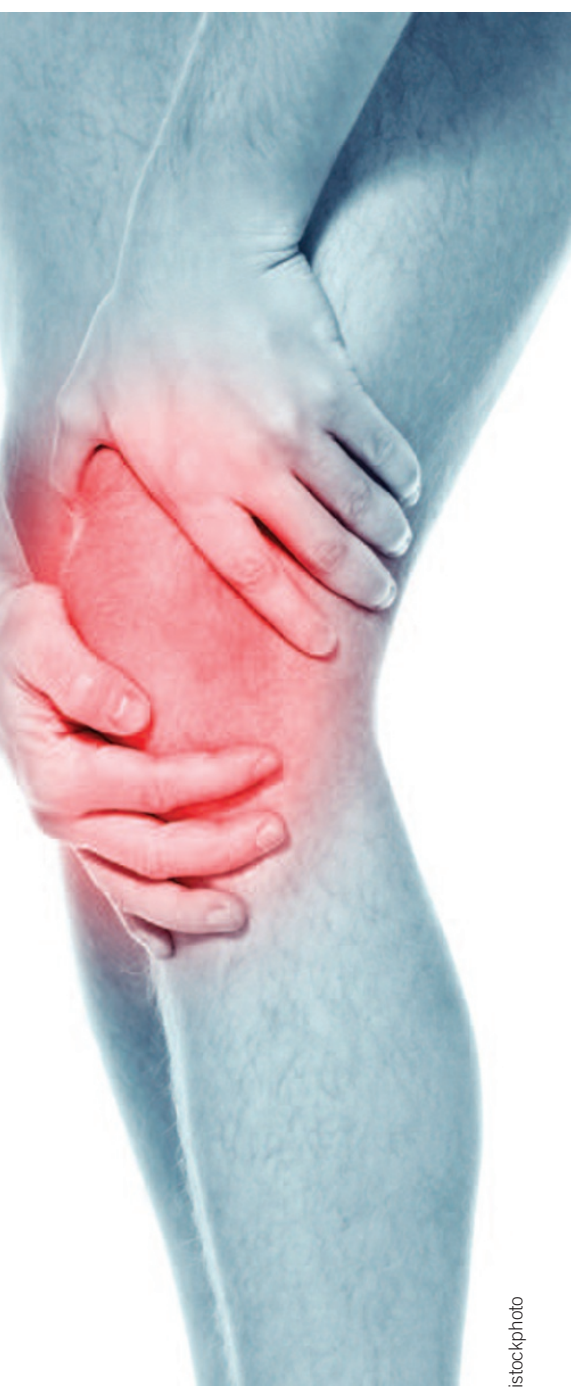

Factor it in: Patients need new options. to do is figure out how to use these drugs better with much closer monitoring."

The therapeutic options for people needing pain relief are sorely lacking. Mild inflammatory pain is typically treated with nonsteroidal anti-inflammatory drugs (NSAIDs) such as aspirin, whereas individuals in more severe pain often take opioids such as morphine. But not everyone responds well to these drugs, and they come with a slew of potential side effects. Thus, pharmaceutical companies have long searched for novel agents to occupy a piece of the $\$ 30$ billion US pain market, and antiNGF agents provided a potential new 'in'.

These drugs, which inhibit a key molecule implicated in pain sensitivity, represented the first biological treatment for pain. Since the first antibody to NGF, Pfizer's tanezumab, entered clinical testing in 2004, four other similar agents have also been tested in people suffering from osteoarthritis-a degenerative joint disorder and the drugs' lead indication-as well as a range of other pain conditions.

In June 2010, however, the FDA placed a clinical hold on tanezumab after study subjects in Pfizer's ongoing phase 3 trials for hip and knee osteoarthritis developed rapidly worsening conditions that necessitated joint replacement surgeries, often in joints other than the ones under evaluation. Six months later, the FDA froze trials for all anti-NGF agents, including Janssen's fulranumab and Regeneron's REGN475, both of which were in phase 2 testing. Only trials in people with terminal cancer were later allowed to continue. Around the same time, MedImmune, a subsidiary of AstraZeneca, also voluntarily delayed development of its antibody, MEDI578, which was in phase 1 trials in the UK, and Abbott finished up a phase 1 study in the Netherlands with its own NGF inhibitor, called PG110, but since then has not taken the drug further.

\section{Taking precautions}

After considering all the data from the three companies in the clinic in the US, the FDA's 21-member panel of arthritis experts recommended that research on the drugs should continue, but with certain safety precautions. Future trials should avoid giving anti-NGF drugs to people also taking NSAIDs because of evidence that such medicines can exacerbate joint problems, the panelists said. In addition, they called for a series of increased surveillance measures to be incorporated into future studies, including more comprehensive radiologic monitoring, and they suggested that drug sponsors should generate more animal data to better understand the neurologic, vascular and bone effects of the drugs.

Because of the risky side effect profiles of anti-NGF drugs, "most of us left the room thinking this was not a class of medicine that will go into widespread use for osteoarthritis or back pain," says Lenore Buckley, a rheumatologist at Virginia Commonwealth University School of Medicine in Richmond who chaired the FDA advisory committee. "Still, this is a class that does seem to have some potency in terms of pain management, and there might be a niche of patients for whom the benefits outweigh the risks, so it's worth looking into further."

George Yancopoulos, chief scientific officer and president of Regeneron Laboratories in Tarrytown, New York, which is co-developing its anti-NGF drug with Sanofi, is taking the panel's recommendations seriously. "Right now it's best to be cautious and assume this is a shared risk of all the molecules," he says. But, unlike the case in his competitors' trials, he notes that none of the hundreds of people with osteoarthritis who enrolled in Regeneron's phase 1 and 2 trials developed the rapidly progressing condition, although he admits that larger trials are still needed to definitively rule out this outcome as a rare possibility. "These are all NGF blockers," he says, "but they are not all the same."

Even so, Natalie Taylor, a pain market analyst at Decision Resources in Burlington, Massachusetts, expects Pfizer's tanezumab, which is the only NGF inhibitor to have entered phase 3 testing, to reach the market first. "It's not a super blockbuster, which we had thought previous to all this safety stuff coming out," Taylor says. But she does forecast global sales for tanezumab to exceed $\$ 5$ billion annually by 2020 for the treatment of osteoarthritis alone, with the other products each earning revenues closer to $\$ 1$ billion per year.

Not all analysts are as bullish, though. “There's so much potential for side effects that it seems like a long shot for these products," says Morningstar's Damien Conover, a pharmaceutical analyst based in Chicago. "We have very low expectations for these products right now."

Elie Dolgin 